\title{
Constraining the co-genesis of Visible and Dark Matter with AMS-02 and Xenon-100
}

\author{
Kazunori Kohri ${ }^{1}$ and Narendra Sahu ${ }^{2}$ \\ 1 Cosmophysics Group, Theory Centre, IPNS, KEK, Tsukuba 305-0801, Japan \\ The Graduate University for Advanced Study (Sokendai), Tsukuba 305-0801, Japan \\ 2 Department of Physics, Indian Institute of Technology Hyderabad, Yeddumailaram 502205, AP, India
}

\begin{abstract}
We study a non-thermal scenario in a two-Higgs doublet extension of the standard model (SM), augmented by an $U(1)_{\mathrm{B}-\mathrm{L}}$ gauge symmetry. In this set up, it is shown that the decay product of a weakly coupled scalar field just above the electroweak scale can generate visible and dark matter (DM) simultaneously. The DM is unstable because of the broken $B-L$ symmetry. The lifetime of $\mathrm{DM}\left(\approx 5 \times 10^{25} \mathrm{sec}\right)$ is found to be much longer than the age of the Universe, and its decay to the SM leptons at present epoch can explain the positron excess observed at the AMS-02. The relic abundance and the direct detection constraint from Xenon-100 can rule out a large parameter space just leaving the $B-L$ breaking scale around $\approx 2-4 \mathrm{TeV}$.
\end{abstract}

\section{INTRODUCTION}

The observed cosmic ray anomalies at PAMELA 1, 2, Fermi [3, 4], H.E.S.S. [5] and recently at AMS-02 [6, 7] (see also [8]) conclusively hint towards a primary source of positron in our Galaxy ${ }^{1}$. This gives rise enough motivation to consider a particle physics based dark matter (DM) models, such as annihilation [11 16] or decay 12, 16 22] of DM, as the origin of positron excess in the cosmic rays ${ }^{2}$.

At present, the relic abundance of DM: $\Omega_{\mathrm{DM}} h^{2} \sim 0.12$, is well measured by the Planck satellite 27]. However, the mechanism that provides its relic abundance is not yet established. Moreover, the origin of tiny amount of visible matter in the Universe which is in the form of baryons with $\Omega_{\mathrm{b}} h^{2} \sim 0.022$ arising from a baryon asymmetry: $n_{B} / n_{\gamma} \sim 6.15 \times 10^{-10}$, has been established by the Planck [27] and the big-bang nucleosynthesis (BBN) measurements [28]. The fact that the DM abundance is about a factor of 5 with respect to the baryonic one might hint towards a common origin behind their genesis.

In fact, both baryon and DM abundances could be produced at the end of inflation, whose origin is usually linked to a scalar field called inflaton [29]. A visible sector inflaton which carries the Standard Model (SM) charges [30] can naturally create a weakly interacting $\mathrm{DM}$, as it happens in the case of Minimal Supersymmetric SM scenarios, see 31. However if the inflaton belongs to a hidden sector, such a SM singlet inflaton, which might as well couple to other hidden sectors, then it becomes a challenge to create the right abundance for both DM and the visible matter.

In this paper we will consider a simple example of any

1 In fact it has been shown earlier that there is a clean excess of absolute positron flux in the cosmic rays at an energy $E \gtrsim 50$ $\mathrm{GeV}$ [9], even if the propagation uncertainty [10] in the secondary positron flux is added to the Galactic background.

2 For astrophysical origins, see Ref. [15, 23- 26] and references therein. generic hidden sector inflaton, which first decays into scalar fields charged under a $U(1)_{B-L}$ gauge group. The subsequent decay of these scalar fields to DM and SM charged leptons generate asymmetry in the visible and DM sectors, which has to be matched with the observed data 27]. The stabilty to DM is provided by the $B-L$ gauge symmetry. We assume that all the above phenomena happens in a non-thermal scenario right above the electroweak scale.

If we assume that $B-L$ is broken above the $\mathrm{TeV}$ scale, then the resulting DM lifetime comes out to be longer than the age of the universe, i.e. $\approx 5 \times 10^{25} \mathrm{sec}$, and it's decay into charged leptons can explain the rising positron spectrum as shown by the AMS-02 data, provided that the DM mass is around $1 \mathrm{TeV}$. Furthermore, we are able to put constraints on the model parameters by the direct detection experiments, such as Xenon-100 [32]. The nulldetetction of DM at Xenon-100 constraints the $B-L$ breaking scale to be around $2-4 \mathrm{TeV}$. The model can be further constrained by the LHC if there is a discovery of an extra $Z^{\prime}$ gauge boson.

The paper is organized as follows. In section-I, we briefly discuss the model. In section-III we provide the mechanism of generating visible and DM simultaneously in a non-thermal set-up. In section-IV we discuss positron anomalies from a decaying DM. In section - $\mathrm{V}$. we discuss compatibility of the DM with the direct detection limits. In section-VI] we conclude our main results.

\section{THE MODEL}

The positron excess seen in PAMELA [1, 2], Fermi [3, 4], AMS-02 [6, 7] experiments hint towards a leptophilic origin of the DM [18, 33]. A simple non-supersymmetric origin of this DM can be explained in a two Higgs doublet extension of the SM with an introduction of an $U(1)_{\mathrm{B}-\mathrm{L}}$ gauge symmetry [18, 34]. We also add three singlet fermions $N_{L}(1,0,-1), \psi_{R}(1,0,-1)$ and $S_{R}(1,0,-1)$ per generation, where the numbers inside the parentheses indicate their quantum numbers under the gauge group 
$S U(2)_{L} \times U(1)_{Y} \times U(1)_{\mathrm{B}-\mathrm{L}}$. We need to check the axialvector anomaly [35], which requires the following conditions to be satisfied for its absence:

$$
\begin{aligned}
& S U(3)_{C}^{2} U(1)_{\mathrm{B}-\mathrm{L}} \quad: \quad 3\left[2 \times \frac{1}{3}-\frac{1}{3}-\frac{1}{3}\right]=0 \\
& S U(2)_{L}^{2} U(1)_{\mathrm{B}-\mathrm{L}} \quad: \quad 2\left[\frac{1}{3} \times 3+(-1)\right]=0 \\
& U(1)_{Y}^{2} U(1)_{\mathrm{B}-\mathrm{L}}: 3\left[2 \times\left(\frac{1}{3}\right)^{2} \times \frac{1}{3}\right] \\
& -3\left[\left(\frac{4}{3}\right)^{2} \times \frac{1}{3}+\left(\frac{-2}{3}\right)^{2} \times \frac{1}{3}\right] \\
& +\left[2(-1)^{2}(-1)-1(-2)^{2}(-1)\right]=0 \\
& U(1)_{Y} U(1)_{\mathrm{B}-\mathrm{L}}^{2}: 3\left[2 \times \frac{1}{3} \times\left(\frac{1}{3}\right)^{2}\right] \\
& -\left[\frac{4}{3} \times\left(\frac{1}{3}\right)^{2}+\left(\frac{-2}{3}\right) \times\left(\frac{1}{3}\right)^{2}\right] \\
& +\left[2(-1)(-1)^{2}-1(-2)(-1)^{2}\right]=0 \\
& U(1)_{\mathrm{B}-\mathrm{L}}^{3}: 3\left[2 \times\left(\frac{1}{3}\right)^{3}-\left(\frac{1}{3}\right)^{3}-\left(\frac{1}{3}\right)^{3}\right] \\
& +\left[2 \times(-1)^{3}-(-1)^{3}\right] \\
& +\left[(-1)^{3}-(-1)^{3}-(-1)^{3}\right]=0
\end{aligned}
$$

where the number 3 in front is the color factor. Thus the model is shown to be free from $B-L$ anomaly and hence can be gauged by introducing an extra gauge boson $Z^{\prime}$. Since $N_{L}$ is a singlet under $S U(2)_{L}$, and it does not carry any charge under $U(1)_{Y}$, its electromagnetic charge is zero. As a result the lightest one can be a viable candidate of the DM. The stability to DM is provided by the gauged $B-L$ symmetry.

However, we also add two massive charged scalars: $\eta^{-}(1,-2,0)$ and $\chi^{-}(1,-2,-2)$ in the particle spectrum such that their interaction in the effective theory breaks lepton number by two units and hence introduces a prolonged lifetime for the lightest $N_{L}$, which is the candidate for DM. As we show later the extremely slow decay of DM can explain the positron excess observed at PAMELA [1], Fermi [4] and recently at AMS-02 [6]. Furthermore, we assume that these particles are produced non-thermally from the cascade decay of the hidden sector inflaton field $\phi(1,0,0)$ just above the EW scale as pictorially depicted in Fig. 1. The particle content and their quantum numbers are summarised in table 1 .

The main interactions are given by the effective Lagrangian:

$$
\begin{aligned}
\mathcal{L}_{\mathrm{eff}} \supseteq \frac{1}{2}\left(M_{N}\right)_{\alpha \beta} \overline{\left(N_{\alpha L}\right)^{c}} N_{\beta L}+\frac{1}{2}\left(M_{\psi}\right)_{\alpha \beta} \overline{\left(\psi_{\alpha R}\right)^{c}} \psi_{\beta R} \\
+\frac{1}{2}\left(M_{S}\right)_{\alpha \beta} \overline{\left(S_{\alpha R}\right)^{c}} S_{\beta R}+\left(g_{S}\right)_{\alpha \beta}\left(\overline{S_{\alpha R}} H \ell_{\beta L}\right) \\
+\left(g_{\psi}\right)_{\alpha \beta}\left(\overline{\psi_{\alpha R}} H \ell_{\beta L}\right)+\mu \eta H_{1} H_{2}+m^{2} \eta^{\dagger} \chi
\end{aligned}
$$

TABLE I: Particle content and their quantum numbers.

\begin{tabular}{|c|c|c|c|}
\hline Particle & $S U(2)_{L} \times U(1)_{Y}$ & $U(1)_{B-L}$ & Mass range \\
\hline$\ell_{L}$ & $(2,-1)$ & -1 & $\mathrm{MeV}$ to $\mathrm{GeV}$ \\
\hline$\ell_{R}^{-}$ & $(1,-2)$ & -1 & $\mathrm{MeV}$ to $\mathrm{GeV}$ \\
\hline$H_{1}, H_{2}$ & $(2,1)$ & 0 & $100 \mathrm{GeV} \rightarrow \mathcal{O}(\mathrm{TeV})$ \\
\hline$\phi$ & $(1,0)$ & 0 & $\mathcal{O}\left(10^{3} \mathrm{TeV}\right)$ \\
\hline$\chi^{-}$ & $(1,-2)$ & -2 & $\mathcal{O}\left(10^{3} \mathrm{TeV}\right)$ \\
\hline$\eta^{-}$ & $(1,-2)$ & 0 & $\mathcal{O}\left(10^{3} \mathrm{TeV}\right)$ \\
\hline$N_{L}$ & $(1,0)$ & -1 & $\mathcal{O}(\mathrm{TeV})$ \\
\hline$\psi_{R}, S_{R}$ & $(1,0)$ & -1 & $\mathcal{O}(\mathrm{TeV})$ \\
\hline
\end{tabular}

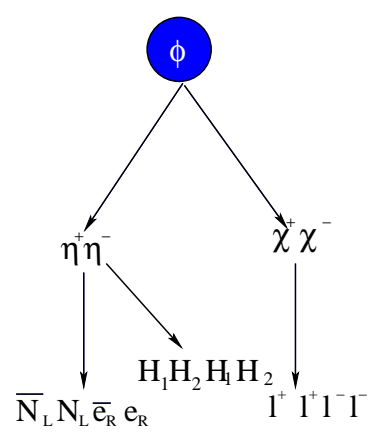

FIG. 1: Decay of hidden sector inflaton to SM degrees of freedom through $\eta$ and $\chi$ fields.

$$
+h_{\alpha \beta} \eta^{\dagger} \overline{N_{\alpha L}} \ell_{\beta R}+f_{\alpha \beta} \chi^{\dagger} \ell_{\alpha L} \ell_{\beta L}+\text { h.c. }
$$

where

$$
m^{2}=\mu^{\prime} v_{\mathrm{B}-\mathrm{L}}, \quad M_{i}=F_{i} v_{\mathrm{B}-\mathrm{L}}
$$

with " $v_{\mathrm{B}-\mathrm{L}}$ " is the vacuum expectation value (vev) of the $U(1)_{\mathrm{B}-\mathrm{L}}$ breaking scalar field which carries $B-L$ charges by two units and $F_{i}$ is the coupling between $B-L$ breaking scalar field and the singlet fermions. In Eq. (11), $H_{1}, H_{2}$ are two Higgs doublets and $\ell_{L}(2,-1,-1)$, $\ell_{R}(1,-2,-1)$ are SM lepton doublet and singlet respectively.

We demand $M_{i}=F_{i} v_{\mathrm{B}-\mathrm{L}}$, with $i=N, S, \psi$, to be of the order of $\mathrm{TeV}$ scale in order to explain the cosmic ray anomalies as discussed in section IV] Since the interactions of $S$ and $\psi$ break $B-L$ by two units, the neutrino mass, after electroweak phase transition, can be generated via the dimension five operators: $\ell \ell H H / M_{S}$ and $\ell_{L} \ell_{L} H H / M_{\psi}$ and is given by:

$$
M_{\nu}=\frac{g_{S}^{2}\langle H\rangle^{2}}{M_{S}}+\frac{g_{\psi}^{2}\langle H\rangle^{2}}{M_{\psi}} .
$$

Taking $M_{S}, M_{\psi} \sim \mathcal{O}(\mathrm{TeV})$, the sub-eV neutrino mass imply $g_{S}, g_{\psi} \sim \mathcal{O}\left(10^{-5}\right)$. Therefore, the decay of $S$ and 


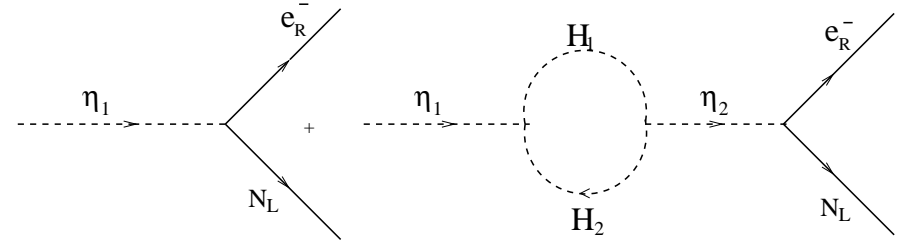

FIG. 2: The interference of tree-level and self-energy correction diagrams which give rise to $\mathrm{CP}$ violation.

$\psi$ can not produce any lepton asymmetry even though their interactions break $B-L$ by two units. Moreover, the number density of these particles are Boltzmann suppressed as the reheat temperature is around $100 \mathrm{GeV}$.

As we will show in section (III), the lepton number conserving decay: $\eta \rightarrow N_{L}+\ell_{R}$ generates visible and DM $\left(N_{L}\right)$ simultaneously. However, note that the interaction between $\eta$ and $\chi$ violates the lepton number by two units. Therefore, the DM is no more stable and decays slowly to SM fields. Since the DM carry a net leptonic charge, it only decays to leptons without producing any quarks. As we will discuss in section (IV) the lifetime of the DM is much longer than the age of the Universe. As a result it could explain the observed positron anomalies at PAMELA [1, 2], Fermi [3, 4] and AMS-02 [6, 7] without conflicting with the antiproton data.

\section{CO-GENESIS OF VISIBLE AND DARK MATTER}

\section{A. Baryon asymmetry}

In this section we explain the details of simultaneously creating the observed baryon asymmetry and the relic abundance of DM in our model. We assume that the hidden sector inflaton $\phi$ with mass $m_{\phi}$ decays into the SM degrees of freedom through $\eta$ and $\chi$ as depicted in Fig. 1. We further assume this gives rise to a reheat temperature:

$$
T_{R} \sim 0.1 \sqrt{\Gamma_{\phi} M_{\mathrm{Pl}}} \gtrsim 100 \mathrm{GeV} .
$$

To generate baryon asymmetry we need $\mathrm{CP}$ violation for which we assume that there exist two $\eta$ fields: $\eta_{1}$ and $\eta_{2}$ of masses $M_{1}$ and $M_{2}$. Since their couplings with $N_{L}$ and $\ell_{R}$ are in general complex, the $B-L$ conserving decay of the lightest one can give rise to $\mathrm{CP}$ violation through the interference of tree level and self energy correction diagrams as shown in the Fig. 2, The $\mathrm{CP}$ violation due to the decay of the lightest $\eta$ can be estimated to be [36],

$$
\epsilon_{L}=\frac{\operatorname{Im}\left[\left(\mu_{1} \mu_{2}^{*}\right) \sum_{\alpha \beta} h_{\alpha \beta}^{1} h_{\alpha \beta}^{2 *}\right]}{16 \pi^{2}\left(M_{2}^{2}-M_{1}^{2}\right)}\left[\frac{M_{1}}{\Gamma_{1}}\right]=-\epsilon_{N_{L}},
$$

where

$$
\Gamma_{1}=\frac{1}{8 \pi M_{1}}\left(\mu_{1} \mu_{1}^{*}+M_{1}^{2} \sum_{i, j} h_{\alpha \beta}^{1} h_{\alpha \beta}^{1 *}\right) .
$$

Now assuming $\mu_{1} \sim \mu_{2} \sim M_{1} \sim M_{2}$ and $h_{\alpha \beta}^{1} \sim h_{\alpha \beta}^{2} \sim$ $\mathcal{O}\left(10^{-2}\right)$ we get from Eqs. (5) and (6) the CP asymmetry $\left|\epsilon_{L}\right|=\left|\epsilon_{N_{L}}\right| \simeq 10^{-5}$.

Since the decay of the lightest $\eta$ does not violate lepton number, so it can not produce a net $B-L$ asymmetry. But it will produce an equal and opposite $B-L$ asymmetry between $N_{L}$ and $\ell_{R}[34,37,38]$. The two asymmetries, which remain isolated from each other before electroweak phase transition, can be given by:

$$
\mathcal{Y}_{\mathrm{B}-\mathrm{L}}=\left.B_{\eta} \epsilon_{L} \frac{n_{\phi}}{s}\right|_{T=T_{R}}=-\mathcal{Y}_{N_{L}}^{\text {asy }}
$$

where $n_{\phi}=\rho_{\phi} / m_{\phi}$ is the inflaton density and $s=$ $\left(2 \pi^{2} / 45\right) g_{*} T^{3}$ is the entropy density. The branching fraction in the above equation is defined by:

$$
B_{\eta}=\frac{\Gamma\left(\phi \rightarrow \eta^{+} \eta^{-}\right)}{\Gamma(\phi \rightarrow \text { all })} .
$$

Using $\left.\rho_{\phi}\right|_{T=T_{R}}=\left(\pi^{2} / 30\right) g_{*} T_{R}^{4}$ in Eq. (7) we get

$$
\mathcal{Y}_{\mathrm{B}-\mathrm{L}}=\frac{3}{4} B_{\eta} \epsilon_{L} \frac{T_{R}}{m_{\phi}}=-\mathcal{Y}_{N_{L}}^{\text {asy }} .
$$

The $B-L$ asymmetry in $\ell_{R}$ can be transformed to $\ell_{L}$ through the lepton number conserving process: $\ell_{R} \ell_{R}^{c} \leftrightarrow$ $\ell_{L} \ell_{L}^{c}$ mediated via the SM Higgs as it remains equilibrium above electroweak phase transition. As a result the $B-L$ asymmetry in the lepton sector can be converted to baryon asymmetry through the $S U(2)_{L}$ sphalerons while leaving an equal and opposite $B-L$ asymmetry in $N_{L}$. The conversion of $B-L$ asymmetry to the baryon asymmetry is obtained by :

$$
\mathcal{Y}_{B}=\frac{24}{92} B_{\eta} \epsilon_{L} \frac{T_{R}}{m_{\phi}} .
$$

For $T_{R} / m_{\phi} \approx 10^{-4}$ and $\epsilon_{L} \approx 10^{-5}$, we can achieve the observed baryon asymmetry $Y_{B} \approx \mathcal{O}\left(10^{-10}\right)$. This leads to the DM to baryon abundance:

$$
\frac{\mathcal{Y}_{N_{L}}^{\text {asy }}}{\mathcal{Y}_{B}}=\frac{92}{32} \text {. }
$$

A crucial point to note here is that the asymmetric component of DM and baryon asymmetry are produced by a non-thermal decay of the $\phi$ decay products, $\eta$ and $\chi$. An obvious danger of washing out this asymmetry comes from the $B-L$ violating process $N_{L} \ell_{R} \rightarrow \ell_{L} \ell_{L}$ through the mixing between $\eta$ and $\chi$. However, this process is suppressed by a factor $\left(m^{2} / M_{\eta}^{2} M_{\chi}^{2}\right)^{2}$ for $m \ll M_{\eta}, M_{\chi}$ and hence it cannot compete with the Hubble expansion parameter at $T_{R} \sim 100 \mathrm{GeV}$. Another lepton number violating process is $\ell_{L} \ell_{L} \rightarrow H H$ mdeiated by $S$ and $\psi$. However, the rate of this process: $\Gamma \sim M_{\nu}^{2} T_{R}^{3} /\langle H\rangle^{4}$ is much 
less than the Hubble expansion parameter at $T_{R} \sim 100$ $\mathrm{GeV}$. As a result the net $B-L$ asymmetry produced by the decay of $\eta$ will be converted to the required baryon asymmetry without suffering any washout.

\section{B. Dark Matter abundance}

Let us now calculate the required DM to baryon ratio:

$$
\frac{\Omega_{N_{L}}}{\Omega_{\mathrm{B}}}=\frac{\mathcal{Y}_{N_{L}}^{\text {asy }}}{\mathcal{Y}_{B}} \frac{M_{N}}{m_{n}}
$$

where $m_{n}$ is the mass of a nucleon, and $M_{N}$ is the Majorana mass of the DM candidate $N_{L}$.

As we discuss in section (IV), $N_{L}$ mass is required to be $\mathcal{O}(\mathrm{TeV})$ to explain the observed cosmic ray anomalies at PAMELA [1, 2], Fermi 3, 4] and recently at AMS02 [6, 7]. However, for $\mathcal{O}(\mathrm{TeV})$ mass of $N_{L}$, Eq. (12) gives $\Omega_{N_{L}}>>\Omega_{B}$. Fortunately this is not be the case, because of the Majorana mass of $N_{L}$ which give rise to rapid oscillation between $N_{L}$ and $N_{L}^{c}$ [39]. As a result the $N_{L}$ asymmetry can be further reduced through the annihilation process: $N_{L} N_{L}^{c} \rightarrow Z_{\mathrm{B}-\mathrm{L}} \rightarrow f \bar{f}$, where $f$ is the SM fermion.

Note that the decay of $\eta$ also give rise to a dominant $B-L$ symmetric abundance of $N_{L}$ and is given by:

$$
\mathcal{Y}_{N_{L}}^{\mathrm{sym}}=\frac{3}{4} B_{\eta} \frac{T_{R}}{m_{\phi}}
$$

which is larger than the asymmetric component $\mathcal{Y}_{N_{L}}^{\text {asy }}$ by five orders of magnitude and hence required further depletion to match with the observed DM abundance.

The total $N_{L}$ abundnace $\mathcal{Y}_{N_{L}}=\mathcal{Y}_{N_{L}}^{\text {sym }}+\mathcal{Y}_{N_{L}}^{\text {asy }} \approx \mathcal{Y}_{N_{L}}^{\text {sym }}$, thus produced non-thermally, can be matched with the observed DM abundance by requiring that the annihilation cross-section:

$$
\langle\sigma|v|\rangle_{\mathrm{ann}} \equiv\langle\sigma|v|\rangle_{\left(N_{L} \overline{N_{L}} \rightarrow Z_{\mathrm{B}-\mathrm{L}} \rightarrow \sum_{f} f \bar{f}\right)} \approx \frac{1}{4 \pi} \frac{M_{N}^{2}}{v_{\mathrm{B}-\mathrm{L}}^{4}},
$$

is larger than the freeze-out value $\langle\sigma|v|\rangle_{F}=2.6 \times$ $10^{-9} \mathrm{GeV}^{-2}$. Note that in the above equation we have used the mass of $Z_{\mathrm{B}-\mathrm{L}}$ boson to be:

$$
M_{Z^{\prime}}=g_{\mathrm{B}-\mathrm{L}} v_{\mathrm{B}-\mathrm{L}},
$$

with $v_{\mathrm{B}-\mathrm{L}}$ is the $B-L$ symmetry breaking scale. In an expanding Universe, the annihilation cross-section (14) has to compete with the Hubble expansion parameter:

$$
H=1.67 g_{*}^{1 / 2} \frac{T^{2}}{M_{\mathrm{pl}}},
$$

and the details of dynamics can be obtained by solving the relevant Boltzmann equations:

$$
\begin{array}{r}
\frac{d n_{\eta}}{d t}+3 n_{\eta} H=-\Gamma_{\eta} n_{\eta}, \\
\frac{d n_{N_{L}}}{d t}+3 n_{N_{L}} H=-\langle\sigma|v|\rangle_{\mathrm{ann}} n_{N_{L}}^{2}+\Gamma_{\eta} n_{\eta} .
\end{array}
$$

If we omit the production term from the thermal bath, i.e., $\Gamma_{\eta} n_{\eta} \rightarrow 0$ in Eq. (17), then $\frac{d n_{N_{L}}}{d t}<<3 n_{N_{L}} H$. In this approximation we obtain,

$$
\mathcal{Y}_{N_{L}} \equiv \frac{n_{N_{L}}}{s} \simeq \frac{3 H}{\langle\sigma|v|\rangle_{\mathrm{ann}} s}
$$

where $s$ is the entropy density. In the above equation $\mathcal{Y}_{N_{L}}$ has to be matched with the observed DM abundance:

$$
\left(\mathcal{Y}_{N_{L}}\right)_{\mathrm{obs}}=4 \times 10^{-13}\left(\frac{1 \mathrm{TeV}}{M_{N}}\right)\left(\frac{\Omega_{\mathrm{DM}} h^{2}}{0.11}\right) .
$$

The matching of Eqs. (18) and (19) at $T=T_{R}$, gives a constraint on the annihilation cross-section to be:

$$
\begin{aligned}
\frac{\langle\sigma|v|\rangle_{\text {ann }}}{\langle\sigma|v|\rangle_{F}}= & 2.74\left(\frac{M_{N}}{3 \mathrm{TeV}}\right)\left(\frac{0.11}{\Omega_{\mathrm{DM}} h^{2}}\right) \\
& \left(\frac{100 \mathrm{GeV}}{T_{R}}\right) .
\end{aligned}
$$

The above equation implies that the annihilation crosssection (14) is a few times larger than the freeze-out value for a reheat temperature of $100 \mathrm{GeV}$. Now combining Eqs. (14) and (20) we can get a constraint on the $B-L$ breaking scale to be

$$
\begin{aligned}
v_{\mathrm{B}-\mathrm{L}}=3.16 \mathrm{TeV} & \left(\frac{\Omega_{\mathrm{DM}} h^{2}}{0.11}\right)^{1 / 4}\left(\frac{M_{N}}{3 \mathrm{TeV}}\right)^{1 / 4} \\
& \times\left(\frac{T_{R}}{100 \mathrm{GeV}}\right)^{1 / 4} .
\end{aligned}
$$

\section{DECAYING DM AND COSMIC RAY ANOMALIES}

The lepton number is violated through the mixing between $\eta$ and $\chi$ as defined by $m^{2} \eta^{\dagger} \chi$. Therefore, the lightest $N_{L}$, which is the candidate of DM, is not stable. We assume that $m<<M_{\eta}, M_{\chi}$. This gives a suppression in the decay rate of DM. In other words the lifetime of DM is longer than the age of the Universe. The only available channel for the decay of lightest $N_{L}$ is three body decay:

$$
N_{L} \rightarrow e_{\alpha R}^{-} e_{\beta L}^{+} \bar{\nu}_{\gamma L}
$$

with $\beta \neq \gamma$. Since the coupling of $\chi$ to two lepton doublets is antisymmetric, i.e., $\beta \neq \gamma$, the decay of $N_{L}$ is not necessarily to be flavor conserving. In particular the decay mode: $N_{L} \rightarrow \tau_{R}^{-} \tau_{L}^{+} \bar{\nu}_{e L}\left(\bar{\nu}_{\mu L}\right)$, violates $L_{e}\left(L_{\mu}\right)$ by one unit while it violates $L=L_{e}+L_{\mu}+L_{\tau}$ by two units.

In the mass basis of $N_{L}$ the lifetime can be estimated to be

$$
\begin{aligned}
\tau_{N}= & 8.0 \times 10^{25} \mathrm{~s}\left(\frac{10^{-2}}{h}\right)^{2}\left(\frac{10^{-8.5}}{f}\right)^{2} \\
& \left(\frac{50 \mathrm{GeV}}{m}\right)^{4}\left(\frac{m_{\phi}}{10^{6} \mathrm{GeV}}\right)^{8}\left(\frac{3 \mathrm{TeV}}{M_{N}}\right)^{5}
\end{aligned}
$$


where we assume that $M_{\eta} \simeq M_{\chi} \approx m_{\phi}$ in order to get a lower limit on the lifetime of $N_{L}$. The prolonged lifetime of $N_{L}$ may explain the current cosmic ray anomalies observed by PAMELA [1, 2], Fermi [3, 4] and recently at AMS-02 [6, 7]. The electron and positron energy spectrum can be estimated by using the same setup as in Ref. [17]. In Figs. 3 and 4 we have shown the integrated electron and positron fluxes in a typical decay mode: $N_{L} \rightarrow \tau^{-} \tau^{+} \bar{\nu}$ up to the maximum available energy $M_{N} / 2$ for two values of decay life-time, namely $\tau_{N}=4 \times 10^{25} \mathrm{sec}$ and $\tau_{N}=5 \times 10^{25}$ sec. ${ }^{3}$. From there it can be seen that the decay of $N_{L}$ can nicely explain the observed cosmic ray excesses at PAMELA, Fermi and at AMS-02. While doing so we assume that the branching fraction in the decay of $N_{L}$ to $\tau^{-} \tau^{+} \bar{\nu}$ is significantly larger than the other viable decay modes: $N_{L} \rightarrow \mu^{-} \mu^{+} \bar{\nu}$ and $N_{L} \rightarrow e^{-} e^{+} \bar{\nu}$.

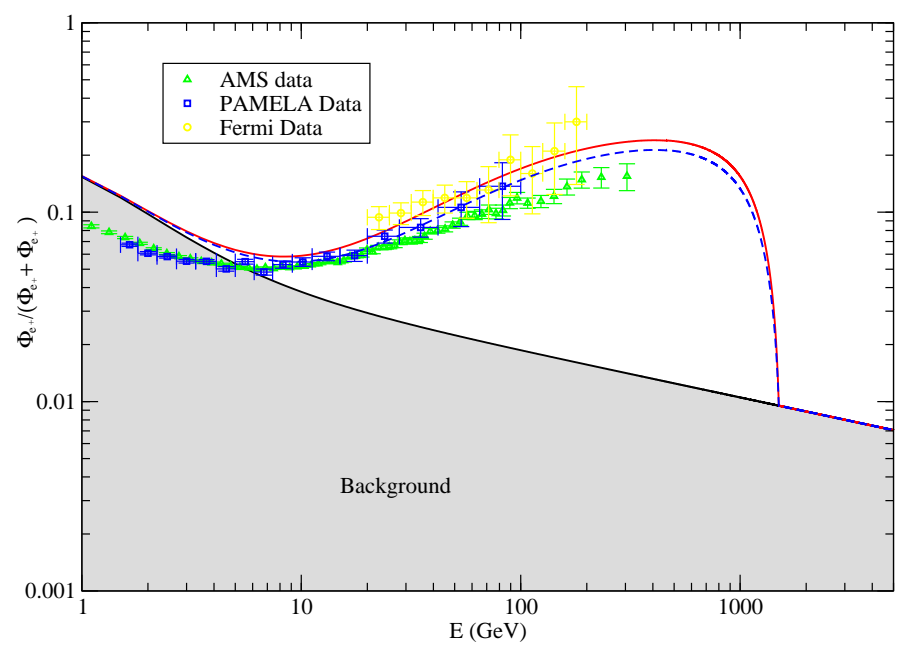

FIG. 3: Positron excess from lightest $N_{L} \rightarrow \tau^{-} \tau^{+} \bar{\nu}$ with $M_{N}=3 \mathrm{TeV}$. The red-solid (top) and Blue-dashed (bottom) lines are shown for $\tau_{N}=4 \times 10^{25} \mathrm{sec}$ and $\tau_{N}=5 \times 10^{25} \mathrm{sec}$ respectively. The fragmentation function has been calculated using PYTHIA [40].

Another potential signature of this scenario is the emission of energetic neutrinos from the Galactic center [41] which can be checked by future experiments such as IceCube DeepCore [42] and KM3NeT [43].

\footnotetext{
3 The constraints on the $\tau^{+}+\tau^{-}$emission modes by gamma-ray emissions from the Galactic center and dwarf spheroidals within the Galaxy depends on the density profile. Since we adopt a cored profile, the constraints are much weaker than those from the Galactic center and dwarf spheroidals [15]
}

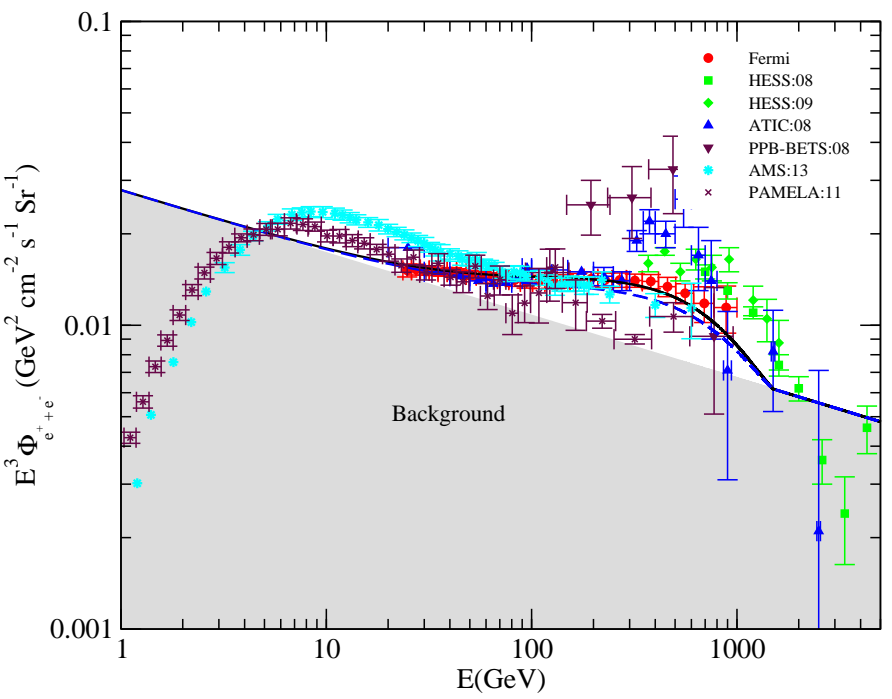

FIG. 4: Total electron plus positron flux from lightest $N_{L} \rightarrow$ $\tau^{-} \tau^{+} \bar{\nu}$ with $M_{N}=3 \mathrm{TeV}$. The Black-solid (top) and Bluedashed (bottom) lines are shown for $\tau_{N}=4 \times 10^{25} \mathrm{sec}$ and $\tau_{N}=5 \times 10^{25}$ sec respectively. The fragmentation function has been calculated using PYTHIA [40].

\section{DIRECT DETECTION OF DARK MATTER AND CONSTRAINTS}

The interaction of $N_{L}$ on the nucleons can give rise to a coherent spin-independent elastic scattering, mediated by the $Z_{\mathrm{B}-\mathrm{L}}$ gauge boson, through $t$-channel process. In the limit of zero-momentum transfer the resulting crosssection is given by:

$$
\begin{aligned}
\sigma_{N_{L} n}= & \frac{\mu_{N_{L} n}^{2}}{64 \pi v_{\mathrm{B}-\mathrm{L}}^{4}}\left(Y_{\mathrm{B}-\mathrm{L}}^{q} Y_{\mathrm{B}-\mathrm{L}}^{N_{L}}\right)^{2} \\
& \left(Z \frac{f_{p}}{f_{n}}+(A-Z)\right)^{2} f_{n}^{2}
\end{aligned}
$$

where $f_{n}$ and $f_{p}$ introduces the hadronic uncertainties in the elastic cross-section and $\mu_{N_{L} n}$ is the reduced mass of DM-nucleon system, given by

$$
\mu_{N_{L} n}=\frac{M_{N} m_{n}}{M_{N}+m_{n}} .
$$

Since $M_{N}>>m_{n}$, one gets $\mu_{N_{L} n} \approx m_{n}$. In Eq. (24), the symbols $Y_{\mathrm{B}-\mathrm{L}}^{q}$ and $Y_{\mathrm{B}-\mathrm{L}}^{N_{L}}$ represent $B-L$ charge of quark and $N_{L}$ respectively. The value of $f_{n}$ vary within a wide range: $0.14<f_{n}<0.66$, as quoted in ref. [44]. Here after we take $f_{n} \simeq \frac{1}{3}$, the central value.

At present the strongest constraint on spinindependent DM-nucleon cross-section is given by Xenon-100, which assumes $f_{p} / f_{n}=1$ with $Z=54$, while $A$ varies between 74 to 80 . This is the case of iso-spin conserving case. For a $3 \mathrm{TeV}$ DM, Xenon-100 gives an upper bound on the DM-nucleon cross-section to be 
$\sigma_{N_{L} n}<\mathcal{O}\left(10^{-43}\right) \mathrm{cm}^{2}$ at $90 \%$ confidence level 32]. From Eq.(24) we can estimate the DM-nucleon cross-section:

$$
\begin{aligned}
\sigma_{N_{L} n}= & 2.15 \times 10^{-43} \mathrm{~cm}^{2}\left(\frac{\mu_{N_{L} n}}{\mathrm{GeV}}\right)^{2} \\
& \left(\frac{5 \mathrm{TeV}}{v_{\mathrm{B}-\mathrm{L}}}\right)^{4} .
\end{aligned}
$$

Thus the $\sigma_{N_{L} n}$ cross-section is in the right order of magnitude and it is compatible with the latest Xenon-100 limit [32]. However, from Eq. (14) we see that for $v_{\mathrm{B}-\mathrm{L}}=5 \mathrm{TeV}$ and $M_{N}=3 \mathrm{TeV}$, the annihilation crosssection: $\langle\sigma|v|\rangle_{\text {ann }}\left\langle\langle\sigma|v|\rangle_{F}=2.6 \times 10^{-9} \mathrm{GeV}^{-2}\right.$. This implies that we get DM abundance more than the observed value and hence $v_{\mathrm{B}-\mathrm{L}} \geq 5 \mathrm{TeV}$ is not allowed. On the other hand, for $v_{\mathrm{B}-\mathrm{L}}<5 \mathrm{TeV}$ we can get right amount of DM abundance. But those values of $v_{\mathrm{B}-\mathrm{L}}$ are not allowed by Xenon-100 constraint as they give large DM-nucleon cross-section. These features can be easily read from Fig. 5 where we have shown the compatibilty of $B-L$ breaking scale with relic abundance (dashed black line) and direct detection constraint (solid red for iso-spin conserving and dot-dashed blue for iso-spin violating) from Xenon-100.

From Eqs.(14) and (24) we see that both the crosssections: $\langle\sigma|v|\rangle_{\text {ann }}$ and $\sigma_{N_{L} n}$ vary inversely as $4^{\text {th }}$ power of $B-L$ breaking scale. Therefore, we need large $\langle\sigma|v|\rangle_{\text {ann }}$ to get the right amount of relic abundance of $\mathrm{DM}$, while small $\sigma_{N_{L} n}$ is required to be compatible with the direct detection limits from Xenon-100. In other words, we need small $v_{\mathrm{B}-\mathrm{L}}$ to get the right amount of relic abundance, while large $v_{\mathrm{B}-\mathrm{L}}$ is required to be compatible with the direct detection limits.

From Fig. 5, we see that for iso-spin conserving case (solid red line) we don't get any value of $v_{\mathrm{B}-\mathrm{L}}$, which is compatible with the relic abundance and the direct detection constraint on DM. However, this constraints can be evaded by considering an iso-spin violating DMnucleon interaction [45] as shown in the Fig. 5 by dotdashed blue line. From there we see that a small window of $B-L$ breaking scale: $v_{\mathrm{B}-\mathrm{L}}=(2.5 \mathrm{TeV}-4 \mathrm{TeV})$ can give $\langle\sigma|v|\rangle_{\text {ann }} \gtrsim\langle\sigma|v|\rangle_{F}$ and $\sigma_{N_{L} n}<\sigma_{\text {Xenon100 }}$ for $M_{N}=$ $3 \mathrm{TeV}$.

Thus we saw that the DM satisfy the direct detection constraints from Xenon-100 only in case of iso-spin violation and within a small window of $B-L$ breaking scale: $v_{\mathrm{B}-\mathrm{L}}=(2.5 \mathrm{TeV}-4 \mathrm{TeV})$. It is worth mentioning that the model though involves many parameters to explain the cosmic ray anomalies from decaying DM, but the relic abundance and the compatibility with direct detection constraints of the latter involves a single parameter, i.e. the $B-L$ breaking scale: $v_{\mathrm{B}-\mathrm{L}}$. In one hand, if $v_{\mathrm{B}-\mathrm{L}}>4 \mathrm{TeV}$, then the annihilation cross-section of DM is smaller than the freeze-out value (see Eq. 14) and hence the model produces large DM abundance. On the other hand, if $v_{\mathrm{B}-\mathrm{L}}<2 \mathrm{TeV}$, then the $\mathrm{DM}$ doesn't satisfy the direct detection constraints from Xenon-100 (see Eq. (24). Note that the above conclusions are independent of other parameters involved in explaining cosmic

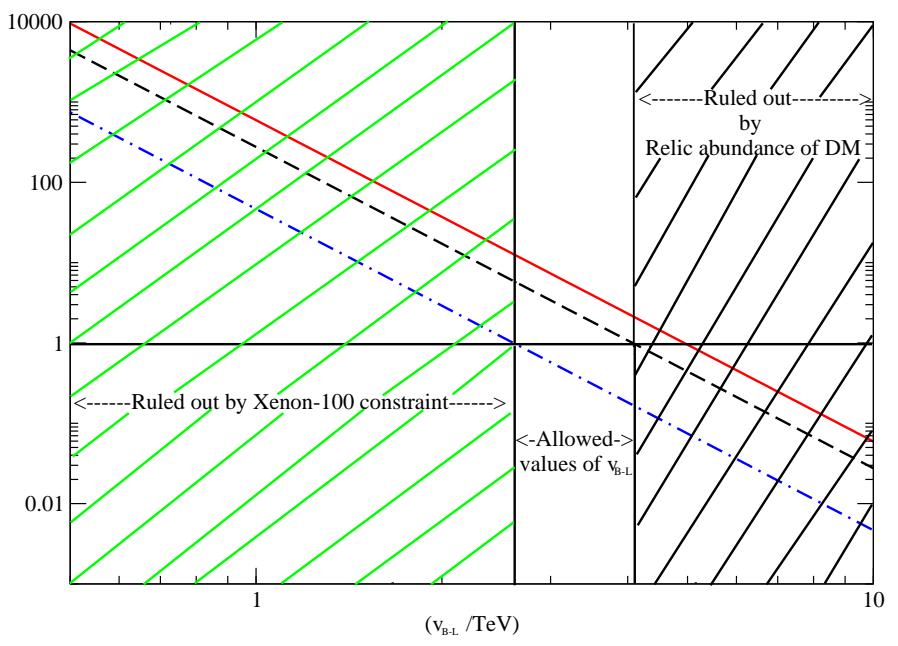

FIG. 5: $\langle\sigma|v|\rangle_{\text {ann }} /\langle\sigma|v|\rangle_{F}$, shown by dashed black and $\sigma_{\mathrm{DMn}} / \sigma_{\mathrm{xenon} 100}$ shown by solid red (iso-spin conserving) and blue dot-dashed (iso-spin violating) as function of $v_{\mathrm{B}-\mathrm{L}}$ for a typical value of the DM mass: $M_{N}=3 \mathrm{TeV}$.

ray anomalies and baryon asymmetry. Therefore, our scenario is strongly constrained in terms of the model parameter and can be checked at the future terrestrial experiments such as Xenon-1T.

\section{CONCLUSIONS}

We studied a non-thermal scenario in a gauged $B-L$ extension of the SM to explain a common origin behind DM abundance and baryon asymmetry. The $B-L$ symmetry is broken at a $\mathrm{TeV}$ scale which gives a Majorana mass to the DM, while the baryon asymmetry is created via lepton number conserving leptogenesis mechanism and therefore it does not depend on the $B-L$ breaking scale. Since the lepton number is violated, the DM is no longer stable and slowly decays into the lepton sector as it carries a net leptonic charge. Since the decay rate of DM is extremely slow, it could explain the positron excess observed at PAMELA, Fermi and recently at AMS02 without conflicting with the antiproton data.

We also checked the compatibility of a $\mathrm{TeV}$ scale DM with the spin-independent DM-nucleon scattering at Xenon-100, which at present gives the strongest constraint on DM-nucleon cross-section. We have found that in the case of iso-spin conserving, the spin independent DM-nucleon cross-section is incompatible with the relic abundance of DM. On the other hand, by assuming the iso-spin violation interaction, we found a small window of $B-L$ breaking scale: $v_{\mathrm{B}-\mathrm{L}}=(2.5 \mathrm{TeV}-4 \mathrm{TeV})$, which can yield right amount of DM abundance while explaining the positron excess. This implies the corresponding $B-L$ gauge boson (i.e. $Z^{\prime}$-gauge boson) is necessarily to be at a TeV scale which can be searched at the LHC. 


\section{ACKNOWLEDGEMENTS}

We thank Anupam Mazumdar, Kazunori Nakayama, Chiara Arina and Julian Heeck for useful discussions. $\mathrm{KK}$ is supported in part by Grant-in-Aid for Scien- tific research from the Ministry of Education, Science, Sports, and Culture (MEXT), Japan, No. 21111006, No. 22244030, and No. 23540327. NS is partially supported by the Department of Science and Technology Grant SR/FTP/PS-209/2011.
[1] O. Adriani et al. [PAMELA Collaboration], Nature 458, 607 (2009) arXiv:0810.4995 [astro-ph]].

[2] O. Adriani et al. [PAMELA Collaboration], Phys. Rev. Lett. 106, 201101 (2011) arXiv:1103.2880 [astroph.HE]].

[3] A. A. Abdo et.al., [Fermi LAT Collaboration], arXiv:0905.0025 [astro-ph.HE];

[4] M. Ackermann et al. [Fermi LAT Collaboration], Phys. Rev. Lett. 108, 011103 (2012) arXiv:1109.0521 [astroph.HE]].

[5] F. Aharonian et al. [H.E.S.S. Collaboration], Astron. Astrophys. 508, 561 (2009) arXiv:0905.0105 [astroph.HE]].

[6] M. Aguilar et al. [AMS Collaboration], Phys. Rev. Lett. 110, no. 14, 141102 (2013).

[7] S. Schael for the AMS Collaboration, Precision measurements of the electron spectrum and the positron spectrum with AMS. Proceedings of the 33rd International Cosmic Ray Conference, Rio de Janeiro, 2-9 July 2013.

[8] J. Chang et al., Nature 456, 362 (2008); S. Torii et al., arXiv:0809.0760 [astro-ph]; F. Aharonian et.al., [HESS Collaboration], arXiv:0905.0105 [astroph.HE]; J. J. Beatty et al., Phys. Rev. Lett. 93 (2004) 241102; M. Aguilar et al. [AMS-01 Collaboration], Phys. Lett. B 646, 145 (2007).

[9] C. Balazs, N. Sahu and A. Mazumdar, JCAP 0907, 039 (2009) arXiv:0905.4302 [hep-ph]].

[10] I. V. Moskalenko and A. W. Strong, Astrophys. J. 493, 694 (1998); T. Delahaye, R. Lineros, F. Donato, N. Fornengo and P. Salati, Phys. Rev. D 77, 063527 (2008); E. A. Baltz and J. Edsjo, Phys. Rev. D 59, 023511 (1999).

[11] L. Bergstrom, J. Edsjo and G. Zaharijas, arXiv:0905.0333 [astro-ph.HE]; M. Cirelli, M. Kadastik, M. Raidal and A. Strumia, arXiv:0809.2409 [hep-ph]; P. Meade, M. Papucci, A. Strumia and T. Volansky, arXiv:0905.0480 [hep-ph]; K. Kohri, J. McDonald and N. Sahu, arXiv:0905.1312 [hep-ph]; D. Hooper and T. M. P. Tait, arXiv:0906.0362 [hep-ph]. P. H. Gu, H. J. He, U. Sarkar and X. Zhang, arXiv:0906.0442 [hep-ph].

[12] K. Hamaguchi, K. Nakaji and E. Nakamura, arXiv:0905.1574 [hep-ph].

[13] J. Kopp, arXiv:1304.1184 [hep-ph].

[14] A. De Simone, A. Riotto and W. Xue, arXiv:1304.1336 [hep-ph].

[15] Q. Yuan, X. -J. Bi, G. -M. Chen, Y. -Q. Guo, S. -J. Lin and X. Zhang, arXiv:1304.1482 [astro-ph.HE].

[16] H. -B. Jin, Y. -L. Wu and Y. -F. Zhou, arXiv:1304.1997 [hep-ph].

[17] K. Ishiwata, S. Matsumoto and T. Moroi, JHEP 0905 , 110 (2009) arXiv:0903.0242 [hep-ph]]. A. Ibarra and D. Tran, JCAP 0902, 021 (2009); S. Shirai, F. Takahashi and T. T. Yanagida, arXiv:0905.3235 [hep-ph]; A. Arvanitaki, S. Dimopoulos, S. Dubovsky, P. W. Gra- ham, R. Harnik and S. Rajendran, arXiv:0904.2789 [hepph]; C. H. Chen, C. Q. Geng and D. V. Zhuridov, arXiv:0905.0652 [hep-ph]; N. Okada and T. Yamada, arXiv:0905.2801 [hep-ph].

[18] K. Kohri, A. Mazumdar, N. Sahu and P. Stephens, Phys. Rev. D 80, 061302 (2009) arXiv:0907.0622 [hep-ph]].

[19] M. Ibe, S. Iwamoto, S. Matsumoto, T. Moroi and N. Yokozaki, arXiv:1304.1483 [hep-ph].

[20] Y. Kajiyama, H. Okada and T. Toma, arXiv:1304.2680 [hep-ph].

[21] M. Ibe, S. Matsumoto, S. Shirai and T. T. Yanagida, arXiv:1305.0084 [hep-ph].

[22] L. Feng and Z. Kang, arXiv:1304.7492 [hep-ph].

[23] D. Hooper, P. Blasi, and P. Dario Serpico, Journal of Cosmology and Astro-Particle Physics 1, 25 (2009); H. Yuksel, M. D. Kistler, and T. Stanev, arXiv:0810.2784 S. Profumo, arXiv:0812.4457; K. Ioka, arXiv:0812.4851 E. Borriello, A. Cuoco and G. Miele, arXiv:0903.1852 [astro-ph.GA]; P. Blasi, arXiv:0903.2794 P. Blasi and P. D. Serpico, arXiv:0904.0871 N. Kawanaka, K. Ioka and M. M. Nojiri, arXiv:0903.3782 [astro-ph.HE]. Y. Fujita, K. Kohri, R. Yamazaki and K. Ioka, arXiv:0903.5298 [astro-ph.HE].

[24] T. Linden and S. Profumo, arXiv:1304.1791 [astroph.HE].

[25] I. Cholis and D. Hooper, arXiv:1304.1840 [astro-ph.HE].

[26] Q. Yuan and X. -J. Bi, arXiv:1304.2687 [astro-ph.HE].

[27] P. A. R. Ade et al. [Planck Collaboration], arXiv:1303.5076 [astro-ph.CO].

[28] J. Beringer et al. (Particle Data Group), Phys. Rev. D86, 010001 (2012).

[29] A. Mazumdar and J. Rocher, Phys. Rept. 497, 85 (2011) arXiv:1001.0993 [hep-ph]].

[30] R. Allahverdi, K. Enqvist, J. Garcia-Bellido and A. Mazumdar, Phys. Rev. Lett. 97, 191304 (2006) hep-ph/0605035. R. Allahverdi, A. Kusenko and A. Mazumdar, JCAP 0707, 018 (2007) hep-ph/0608138. R. Allahverdi, K. Enqvist, J. GarciaBellido, A. Jokinen and A. Mazumdar, JCAP 0706, 019 (2007) hep-ph/0610134.

[31] R. Allahverdi, B. Dutta and A. Mazumdar, Phys. Rev. Lett. 99, 261301 (2007) arXiv:0708.3983 [hepph]]. R. Allahverdi, B. Dutta and A. Mazumdar, Phys. Rev. D 75, 075018 (2007) hep-ph/0702112 [HEP-PH]]. C. Boehm, J. Da Silva, A. Mazumdar and E. Pukartas, Phys. Rev. D 87, 023529 (2013) arXiv:1205.2815 [hep$\mathrm{ph}]$.

[32] E. Aprile et al. [XENON100 Collaboration], Phys. Rev. Lett. 109, 181301 (2012) arXiv:1207.5988 [astroph.CO]].

[33] P. J. Fox and E. Poppitz, Phys. Rev. D 79, 083528 (2009) arXiv:0811.0399 [hep-ph]];

B. Kyae, JCAP 0907, 028 (2009) arXiv:0902.0071 [hep$\mathrm{ph}]$; 
X. -J. Bi, X. -G. He and Q. Yuan, Phys. Lett. B 678, 168 (2009) arXiv:0903.0122 [hep-ph]];

A. Ibarra, A. Ringwald, D. Tran and C. Weniger, JCAP 0908, 017 (2009) arXiv:0903.3625 [hep-ph]].

[34] N. Sahu and U. Sarkar, Phys. Rev. D 78, 115013 (2008).

[35] C.Q. Geng and R.E. Marshak, Phys. Rev. D 39,693 (1989); X.G. He,G.C. Joshi and R.R. Volkas, Phys. ReV. D 41, 278 (1990).

[36] E. Ma and U. Sarkar, Phys. Rev. Lett. 80, 5716 (1998) arXiv:hep-ph/9802445.

[37] J. McDonald, N. Sahu and U. Sarkar, JCAP 0804, 037 (2008).

[38] In case of "Dirac Leptogenesis" there is no need of Lepton number violation. See for instance K. Dick, M. Lindner, M. Ratz and D.Wright, Phys. Rev. Lett.84, 4039 (2000).

[39] C. Arina and N. Sahu, Nucl. Phys. B 854, 666 (2012) arXiv:1108.3967 [hep-ph]];

M. Cirelli, P. Panci, G. Servant, G. Zaharijas, JCAP 1203 (2012) 015 arXiv:1110.3809;

C. Arina, J. -O. Gong and N. Sahu, Nucl. Phys. B 865, 430 (2012) arXiv:1206.0009 [hep-ph]];
C. Arina, R. N. Mohapatra and N. Sahu, Phys. Lett. B 720, 130 (2013) arXiv:1211.0435 [hep-ph]].

[40] T. Sjostrand, S. Mrenna and P. Skands, JHEP 0605, 026 (2006).

[41] J. Hisano, M. Kawasaki, K. Kohri and K. Nakayama, Phys. Rev. D79 (2009) 043516; J. Hisano, K. Nakayama and M. J. S. Yang, arXiv:0905.2075 [hep-ph]; J. Liu, P. f. Yin and S. h. Zhu, arXiv:0812.0964 [astro-ph].

[42] D. F. Cowen [IceCube Collaboration], J. Phys. Conf. Ser. 110, 062005 (2008).

[43] A. Kappes and f. t. K. Consortium, arXiv:0711.0563 [astro-ph].

[44] R. Koch, Z.Physik C 15161 (1982) ; J. Gasser, H. Leutwyler and M. E. Sainio, Phys. Lett. B 253260 (1991) ; M. M. Pavan, R. A. Arndt, I. I. Strakovski and R. L. Workman, PiN Newslett. 16110 (2002) ; A. Bottino, F. Donato, N. Fornengo and S. Scopel, Phys. ReV. D 78 083520 (2008), arXiv:0806.4099.

[45] J. L. Feng, J. Kumar, D. Marfatia and D. Sanford, Phys. Lett. B 703, 124 (2011) arXiv:1102.4331 [hep-ph]]. 